\title{
ReaR

\section{Técnica de reemplazo renal en la insuficiencia renal aguda del paciente crítico: novedades e incertidumbres}

Artículo original: Bagshaw SM, Darmon M, Ostermann M, Finkelstein O, Wald R, Tolwani AJ et al. Current State of the art for renal replacement therapy in critically ill patients with acute kidney injury. Intensive Care Med 2017;43:841-854 (

Jiménez Vizuete JM, Monsalve Naharro JA, García Blasco 0.

\section{Hospital General Universitario de Albacete.}

\section{Resumen}

La insuficiencia renal aguda (IRA) es una complicación frecuente en los pacientes que ingresan en la Unidad de Cuidados Intensivos (UCI). Suele aparecer asociada a otras disfunciones orgánicas en el contexto de un síndrome de disfunción multiorgánica.

Con frecuencia el manejo de la IRA en el paciente crítico requiere terapias de reemplazo renal (TRR) que pretenden sustituir la filtración renal alterada.

Desde la aparición de las TRR hace más de 30 años, ha sido notable el avance en los conocimientos adquiridos y las mejoras en el manejo de los pacientes con IRA. Sin embargo la mortalidad de la IRA sigue siendo elevada y existen muchas controversias y poca claridad a la hora de aplicar las TRR, como momento de inicio y de retirada, la modalidad de tratamiento, la dosis, el tipo de anticoagulación etc. En los últimos años numerosos trabajos han intentado aclarar la falta de consenso existente en muchos de estos aspectos.

\section{Introducción}

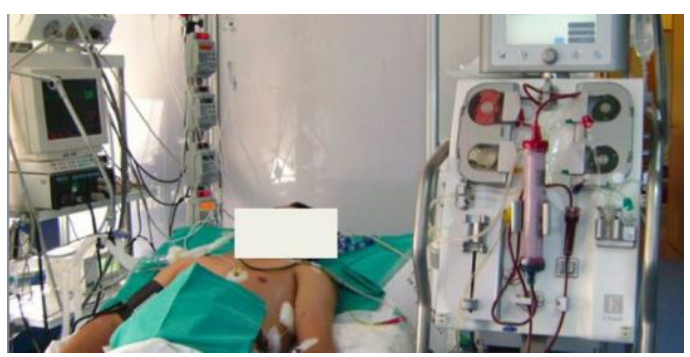

La insuficiencia renal aguda (IRA) es una complicación frecuente en los pacientes que ingresan en la Unidad de Cuidados Intensivos (UCI). Suele aparecer asociada a otras disfunciones orgánicas en el contexto de un síndrome de disfunción multiorgánica. Con frecuencia el manejo de la IRA en el paciente crítico requiere terapias de reemplazo renal (TRR) que pretenden sustituir la filtración renal alterada. Desde la aparición de las TRR hace más de 30 años, ha sido notable el avance en los conocimientos adquiridos y las mejoras en el manejo de los pacientes con IRA. Sin embargo la mortalidad de la IRA sigue siendo elevada (1) y existen muchas controversias y poca claridad a la hora de aplicar las TRR, como momento de inicio y de retirada, la modalidad de tratamiento, la dosis, el tipo de anticoagulación etc. En los últimos años numerosos trabajos han intentado aclarar la falta de consenso existente en muchos de estos aspectos.

\section{Resumen}

\section{$\underline{\text { Objetivos }}$}

Este trabajo es una revisión que pretende actualizar y resumir los recientes avances en las TRR para la IRA que aparece en el paciente crítico. 


\section{Material y métodos}

La metodología y formulación de las recomendaciones se basa en el análisis y revisión no sistemática de los estudios más relevantes (ensayos clínicos aleatorizados, metaanálisis y otros de menor calidad metodológica) relacionados con los siguientes aspectos de las TRR: momento de inicio, catéteres, modalidad de tratamiento, control de solutos, control de volumen, anticoagulación, retirada de las TRR y evolución a largo plazo.

\section{$\underline{\text { Resultados y Conclusiones }}$}

Un resumen de las recomendaciones de esta revisión se presenta en la Tabla 1. La utilización de las TRR como tratamiento de soporte de la IRA en el paciente crítico es cada vez mayor, y existe la necesidad de clarificar diversos aspectos relacionados con su uso, especialmente el momento de inicio, para lo que están en marcha algunos ensayos clínicos. En este contexto es también relevante la falta de indicadores de calidad y de trabajos homogéneos, rigurosos y pragmáticos que nos ayuden a mejorar los resultados en los pacientes críticos que desarrollan IRA y que requieren TRR.
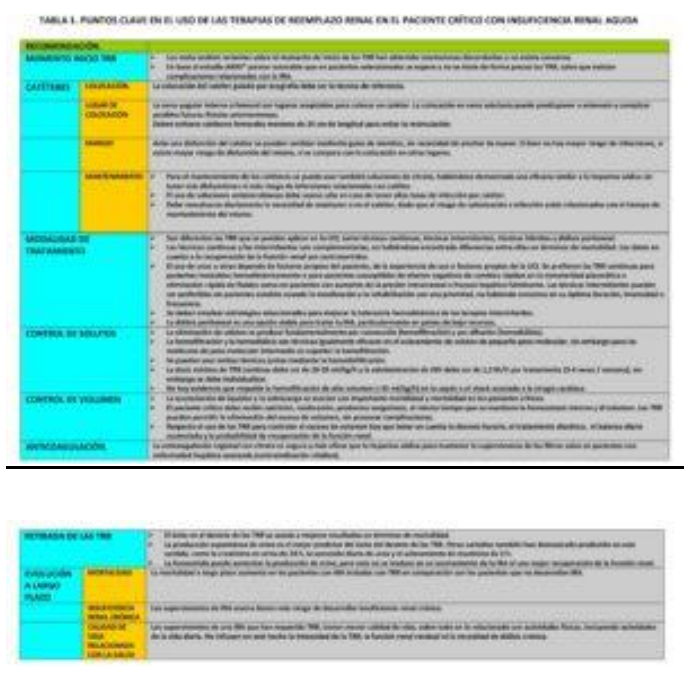

\section{Comentarios}

Esta revisión ofrece una interesante puesta al día de los aspectos más relevantes a tener en cuenta en el uso de las TRR en el ámbito de los cuidados críticos. A pesar del uso extendido de las TRR en las UCI, y de los trabajos publicados al respecto, es destacable que todavía desconocemos cuál es su óptimo momento de inicio en la IRA, si bien un reciente metaanálisis no encontró diferencias en la mortalidad entre un inicio precoz o un inicio tardío (2). Quedamos a la espera de los resultados de dos ensayos clínicos actualmente en desarrollo: el ensayo STARRT-AKI (Standard vs. Accelerated Initiation of RRT in Acute Kidney Injury) y el ensayo IDEAL-UCI (Initiation of Dialysis EArly Versus deLayed in Intensive Care Unit) en el subgrupo de pacientes críticos con shock séptico.

Es destacable la falta de uniformidad de los estudios a los que hace referencia esta revisión. Existe una amplia heterogenidad en variables como los criterios de inclusión o el momento de inicio, por ejemplo mediante el uso de criterios de tiempo (6h, 8h ó 12h) en unos trabajos, o criterios analíticos en otros (niveles de urea o creatinina séricos). También es llamativo el intervalo de tiempo empleado, para unos definido como precoz en las primeras $6 \mathrm{~h}$ y para otros en las primeras 12h. Es evidente que hay mucha diferencia de tiempo para un paciente crítico que evoluciona muy rápidamente $y$ en el que el factor tiempo puede ser crucial para el pronóstico de la enfermedad.

Con respecto a la técnica, destacamos que la ecografía debe ser el método a utilizar en la colocación de catéteres venosos centrales o arteriales ya que disminuye el riesgo de complicaciones (3), sin embargo no hay que olvidar que requiere aparataje, aprendizaje y es 
operador dependiente. En cuanto al cambio de catéteres mediante una guía ante una disfunción, estamos en contra de un intercambio con guía, debido a que el problema puede residir en la vena $y$ no en el catéter, $y$ a que ha demostrado un riesgo de disfunción de hasta 3 veces superior con respecto a una nueva punción en otra localización. Queremos resaltar como algo novedoso los posibles beneficios del uso local de citrato como anticoagulante frente a la heparina sódica para el mantenimiento de los catéteres.

Pensamos que en el paciente crítico con IRA no se debe plantear la disyuntiva de modalidades continuas o intermitentes de TRR, si no hacer un enfoque dinámico, adaptando la técnica a la situación clínica y hemodinámica del paciente en cada momento. En este contexo, técnicas como la hemodiálisis intermitente debe ser una TRR más que se debe ofrecer a los pacientes en la UCI, dado que no hay diferencias en cuanto a la mortalidad ni recuperación de la función renal (4), y a que puede ser beneficiosa con respecto al uso de terapias continuas en algunos pacientes que puedan beneficiarse de una movilización y una rehabilitación al discontinuar la TRR. En cualquier caso no cabe duda de que la disponibilidad de unas u otras técnicas o la experiencia de la UCI van a influir a la hora de

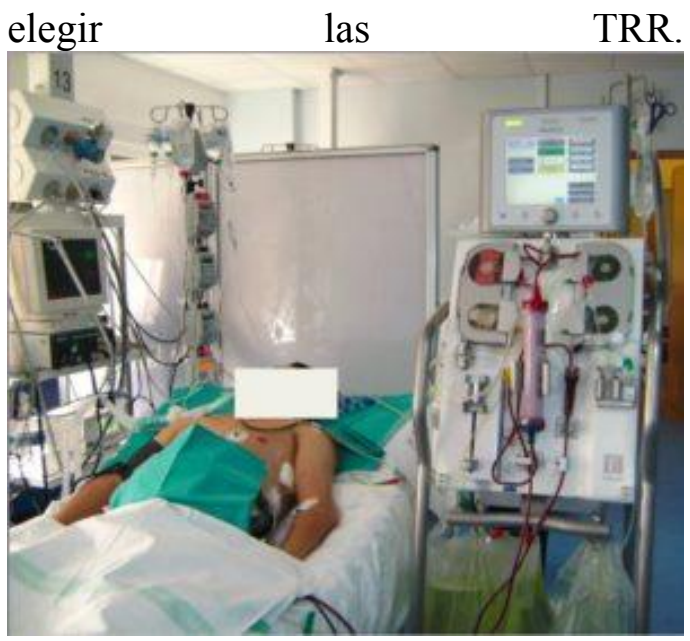

Aspecto importante es la utilidad de las
TRR para mantener un balance hídrico adecuado en nuestros pacientes críticos. Es bien conocida la relación entre balance hídrico positivo y la mortalidad (5).

Queremos destacar a la anticoagulación regional con citrato como forma de anticoagulación de elección del sistema de TRR en base a las pruebas disponibles. Ha demostrado, comparado con la anticoagulación clásica con heparina sódica, menor incidencia de sangrado, menor número de filtros usados $y$ un ahorro económico significativo (6).

Aunque es muy extendido el empleo de la furosemida en el manejo de la IRA en la mayoría de las UCI, sobre todo cuando se retiran momentáneamente las TRR, parece que no acorta la duración de la IRA ni mejora la recuperación de la función renal.

Nos parece acertado que esta revisión haya incluido un apartado sobre la evolución a largo plazo del paciente con IRA sometido a TRR, con referencia a la mortalidad, desarrollo de insuficiencia renal crónica o calidad de vida de los pacientes. Quedan por esclarecerse numerosas incertidumbres y es deseable que tanto el diseño de los futuros estudios como la práctica clínica diaria sean homogéneos y guiados uniformemente a través de recomendaciones encaminadas a mejorar tanto la seguridad como la morbimortalidad de nuestros pacientes críticos.

\section{Bibliografía}

1) Seller-Pérez G, Más-Font $S$, Pérez-Calvo $C$, Villa-Díaz P, Celaya-López M, HerreraGutiérrez ME. Acute kidney injury: Renal disease in the ICU. Med Intensiva 2016;40:374382. (PubMed) (HTML)

2) Bhalt GC, Das RR. Early versus late initiation of renal replacement therapy in patients with acute kidney injury: a systematic 
review and meta-analysis of randomized controlled trials. BMC Nephrol 2017; 18:78. Doi: 10.1186/s12882-017-0486-9. (ePub)

3) Powell JT, Mink JT, Nomura JT, Levine BJ, Jasani N, Nichols WL et al. Ultrasound guidance can reduce adverse events during femoral central venous cannulation. J Emerg Med 2014;46:519-24. (PubMed) (HTML)

4) Nash DM, Przech S, Wald R, O'Reilly D. Systematic review and meta-analysis of renal replacement therapy modalities for acute kidney injury in the intensive care unit. J Crit Care 2017;41:138-144. (PubMed) (HTML

5) Wu B, Sun J, Liu S, Yu X, Zhu Y, Mao H et al. Relationship among mortality of patients with acute kidney injury after cardiac surgery, fluid balance and ultrafiltration of renal replacement therapy: an observational study. Blood Purif 2017;44:32-39. (․ㅡstract)
6) Ricci D, Panicali L, Facchini MG, Mancini E. Citrate anticoagulation during continuous renal replacement therapy. Contrib Nephrol

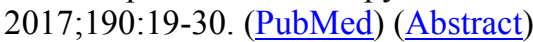

Correspondencia al autor

José María Jiménez Vizuete

josemvizuete@gmail.com

Jefe de Sección. Unidad de Cuidados Intensivos de Anestesia.

Servicio de Anestesiología, Reanimación y Terapéutica del Dolor. Sección de Cuidados Intensivos de la SEDAR.

Hospital General Universitario de Albacete.

Aceptado para blog en septiembre de 2017. 